www.jmscr.igmpublication.org

Impact Factor 5.84

Index Copernicus Value: 83.27

ISSN (e)-2347-176x ISSN (p) 2455-0450

crossref DOI: https://dx.doi.org/10.18535/jmscr/v5i8.79

Journal Of Medical Science And Clinical Research

\title{
Analysing the Role of Computed Tomography in the Diagnosis of Acute Pancreatitis and in Grading its Severity
}

\author{
Authors
}

\section{Ashwin Kumar $\mathbf{S}^{1}$, Daisy Joseph $\mathbf{V}^{2}$, Nandhini $\mathbf{S}^{3}$, Vivek $\mathbf{K}^{4}$}

${ }^{1}$ Formerly Senior Resident, Department of Radiodiagnosis, Govt Medical College, Kottayam

${ }^{2}$ Assistant professor, Department of Radiodiagnosis, Govt Medical College, Kottayam

${ }^{3}$ Senior Resident, Department of Radiodiagnosis, Govt Medical College, Kottayam

${ }^{4}$ Junior Resident, Department of Radiodiagnosis, Govt Medical College, Kottayam

Corresponding Author

\section{Daisy Joseph V}

Email:dsphilipc@gmail.com

\begin{abstract}
Background: Acute pancreatitis is one of the most common gastroenterological disease. Alcoholism and biliary tract disease (gallstones) account for approximately $90 \%$ of cases. The incidence in Europe and United states ranges from 20 to over 70 per 100,000 population. The present study aims to evaluate CT manifestations of acute pancreatitis and to grade its severity using modified CT severity index score.

Materials and methods: Designed as descriptive study among the patients clinically suspected to have acute pancreatitis in the department of RA diodiagnosis, Kottayam. CT findings were evaluated an given scores in even numbers using modified CT severity index score in 100 patients.

Results: Out of 100 patients, CT shows $88 \%$ had acute pancreatitis, $4 \%$ had chronic pancreatitis without acute CT findings and 8\% had normal CT appearance. Using modified CT severity score, 36\% had mild disease, 53\% had moderate and only $11 \%$ had severe disease. Peripancreatic fat stranding was the most common finding followed by size of the pancreas.

Conclusion: CT plays a very important role in the early diagnosis of acute pancreatitis and to grade the extent and severity of the disease and thus helpful in better management of the patients.
\end{abstract}

Keywords: Acute pancreatitis, computed tomography, modified CT severity index score.

\section{Introduction}

Acute pancreatitis was first described by Dutchman Nicolas Tulp in $1652 .{ }^{1}$ It is one of the most common gastroenterological disease. Alcoholism and biliary tract disease (gallstones) account for approximately $90 \%$ of cases. The incidence in Europe and United states ranges from 20 to over 70 per 100,000 population. ${ }^{2,3}$

Computed tomography gives a global picture of the pathology and complications of acute pancreatitis. CT has the advantages over other imaging techniques that it is accurate in establishing the diagnosis, and in assessing for complications. CT is reproducible and reliable from a clinician's perspective; and it is relatively inexpensive and widely available. ${ }^{4} \mathrm{CT}$ has four major indications in patients with suspected or known acute pancreatitis: to establish the diagnosis and exclude other serious intraabdominal conditions; to assess the severity of the pancreatitis; to detect pancreatic and extrapancreatic complications, such as pancreatic 
necrosis, abscess formation, and involvement of surrounding solid organs, vascular structures or gastrointestinal tractomputed tomography helps in early diagnosis of acute pancreatitis and its complicaions. The reported CT sensitivity for the diagnosis of acute pancreatitis ranges from $77 \%$ to $92 \%$. The usefulness of CT is further supported by its high specificity. In most series, there are few false positive findings and CT specificity as high as $100 \%^{5}$ has been reported. In addition by examining the entire abdomen, CT can reveal a variety of other abdominal conditions in patients with clinically suspected acute pancreatitis.

Sonography is considered a useful procedure in the evaluation of patients presenting with abdominal pain in which the differential diagnosis includes acute pancreatitis. However, due to overlying bowel gas, the technique fails to evaluate the pancreas in approximately $20 \%$ of patients. US may be helpful in the follow-up of fluid collections and pseudocysts in selected cases. US may also be used to guide and guide interventional procedures. Ultrasound, despite its multi-planar capability and real-time features, is found to have low sensitivity. Hence CT is the preferred over Ultrasound in Acute pancreatitis. ${ }^{6}$

Limitations in the CT diagnosis of acute pancreatitis are related to suboptimal examinations resulting from poor technique, lack of intravenous contrast medium, or inability of the patient to cooperate. The incidence of normal CT scans in these persons has been estimated as $14-$ $28 \%$. But it is not well established because surgical or pathological correlation is lacking.

Table 1: Modified CT severity index ${ }^{7}$

\begin{tabular}{l|c}
\hline prognostic indicator & points \\
\hline $\begin{array}{l}\text { Pancreatic inflammation } \\
\text { Normal pancreas }\end{array}$ & 0 \\
$\begin{array}{l}\text { Intrinsic pancreatic abnormalities (bulky edematous pancreas) } \\
\text { with or without inflammatory changes in peripancreatic fat }\end{array}$ & 2 \\
$\begin{array}{l}\text { (peripancreatic fat strandings) } \\
\text { Pancreatic or peripancreatic fluid collection or peripancreatic fat }\end{array}$ & 4 \\
necrosis & \\
$\begin{array}{l}\text { Pancreatic necrosis } \\
\text { None }\end{array}$ & 0 \\
$<30 \%$ & 2 \\
$>30 \%$ & 4 \\
$\begin{array}{l}\text { Extrapancreatic complications } \\
\text { (one or more of pleural effusion, ascites, vascular }\end{array}$ \\
$\begin{array}{l}\text { complications, parenchymal complications (infected necrosis , } \\
\text { abscess), or gastrointestinal tract involvement) }\end{array}$ \\
\hline
\end{tabular}

Modified CT severity index (Table 1) incorporated features reflecting organ failure and extra-pancreatic complications and would be useful for predicting outcomes more accurately.

\section{Materials and Methods}

A descriptive cross sectional study was conducted in 100 patients, clinically suspected to have acute pancreatitis in the department of Radiodiagnosis, Kottayam for about 9 months from January 2013 to September 2013. Patients with history of allergic reactions to iodinated contrast agents and patients not willing to take part in the study were excluded from the study.

\section{Study Instrument}

Non contrast and contrast enhanced computed tomography of abdomen and pelvis were taken in a 4 slice Toshiba asteion spiral CT machine.

\section{Study Procedure}

After obtaining ethical clearance and consent from the patient, non contrast images were acquired as $5 \mathrm{~mm}$ sections from the level of xiphisternum to pubic symphysis. Post contrast images were acquired in the portal venous phase, 65 seconds after the administration of $100-150 \mathrm{ml}$ of nonionic iodinated contrast agent (iohexol) by intravenous route. Post contrast images were acquired as $3 \mathrm{~mm}$ sections of upper abdomen and $5 \mathrm{~mm}$ sections of rest of the abdomen.

\section{Data Collection and Analysis}

All the images were reconstructed into sagittal and coronal planes using multiplanar reconstruction. Computed tomographic imaging findings (Pancreatic size, enhancement pattern, presence or absence of intrapancreatic / extra pancreatic fluid collection, presence or absence of pancreatic necrosis, percentage of pancreatic necrosis, presence or absence of peripancreatic fat necrosis, pancreatic duct size, presence or absence of calcifications, presence or absence of extrapancreatic involvement or pancreatic 
parenchymal complications) of each patient were recorded in the case report form (profoma) and entered in MS Excel.. Based on these, patients were given scores in even numbers, ranging from $0-10$ using modified CT severity index. Patients given scores using modified CT severity index were further categorized into three groups as mild, moderate and severe. Percentage of mortality in each category of mild, moderate and severe were recorded.

Data obtained were analyzed using descriptive statistics which includes measure of dispersion and measure of central tendencies.

\section{Results}

The final sample size was 100 and these cases were included in the statistical evaluation of the study. $7 \%$ were in the age group $20-29$ yrs, $23 \%$ in the age group $30-39 \mathrm{yrs}, 35 \%$ in the age group $40-49$ yrs, $28 \%$ in the age group $50-59$ yrs, $5 \%$ in the age group $60-69 y$ rs and $1 \%$ in the age group $70-79 y r s$. Mean age of the sample is 45 yrs. Youngest patient is of 22 yrs age and Eldest patient is of 70yrs age.

Out of 100 cases, $80 \%$ of cases were male and $20 \%$ were female (Table 2). $78 \%$ cases had increase in size of pancreas, $5 \%$ showed atrophic pancreas, $17 \%$ had normal size pancreas (Table 3). Peripancreatic fat strandings were seen in $80 \%$ of cases and absent in $20 \%$ of cases (Table 4). Pancreatic necrosis was seen in $15 \%$ of cases (11\% had $<30 \%$ necrosis, $4 \%$ had $>30 \%$ necrosis). Pancreatic necrosis was absent in $85 \%$ of cases (Table 5). Peripancreatic fat necrosis was seen in only $6 \%$ of study sample. $94 \%$ of the cases did not have peripancreatic fat necrosis (Table 6).

Based on modified CT severity index, 36\% had mild pancreatitis, $53 \%$ had moderate pancreatitis and $11 \%$ had severe pancreatitis (Table 7).

$8 \%$ had normal CT appearance, 56\% had acute pancreatitis without complications, $2 \%$ had complicated acute pancreatitis, 30\% had acute exacerbation of chronic pancreatitis and $4 \%$ had chronic pancreatitis without CT evidence of acute exacerbation (Table 8).

Table 2: Frequency distribution of study sample based on sex

\begin{tabular}{|l|c|}
\hline Sex & Frequency \\
\hline male & 80 \\
\hline Female & 20 \\
\hline
\end{tabular}

Table 3: Frequency distribution of study sample based on the pancreatic size

\begin{tabular}{|l|l|l|}
\hline Pancreatic size & Frequency & Percentage \\
\hline Increased & 78 & $78 \%$ \\
\hline Normal & 17 & $17 \%$ \\
\hline Atrophic & 5 & $5 \%$ \\
\hline
\end{tabular}

Table 4: Frequency distribution of study sample based on Peripancreatic fat stranding

\begin{tabular}{|l|c|c|}
\hline Peripancreatic fat stranding & Frequency & Percentage \\
\hline present & 80 & $80 \%$ \\
\hline absent & 20 & $20 \%$ \\
\hline
\end{tabular}

Table 5: Frequency distribution of study sample based on Pancreatic necrosis

\begin{tabular}{|l|c|c|c|}
\hline \multicolumn{2}{|c|}{ Pancreatic necrosis } & Frequency & Percentage \\
\hline \multicolumn{2}{|c|}{ No necrosis } & 85 & $85 \%$ \\
\hline \multirow{2}{*}{ Necrosis } & $<30 \%$ & 11 & $11 \%$ \\
\cline { 2 - 4 } & $>30 \%$ & 4 & $4 \%$ \\
\hline
\end{tabular}

Table 6 : Frequency distribution of study sample based on Peripancreatic necrosis

\begin{tabular}{|l|c|c|}
\hline Peripancreatic necrosis & Frequency & Percentage \\
\hline Absent & 94 & $94 \%$ \\
\hline Present & 6 & $6 \%$ \\
\hline
\end{tabular}

Table 7 : Frequency distribution of Study sample according to severity based on modified CT severity score

\begin{tabular}{|l|c|c|}
\hline Severity & Frequency & Percentage \\
\hline Mild & 36 & $36 \%$ \\
\hline Moderate & 53 & $53 \%$ \\
\hline Severe & 11 & $11 \%$ \\
\hline
\end{tabular}

Table 8 : Frequency distribution of study sample based on computed tomographic diagnosis

\begin{tabular}{|l|c|}
\hline CT diagnosis & Frequency \\
\hline Normal CT appearance & 8 \\
\hline Acute pancreatitis without complication & 56 \\
\hline Complicated acute pancreatitis & 2 \\
\hline Acute exacerbation of chronic pancreatitis & 30 \\
\hline $\begin{array}{l}\text { Chronic pancreatitis without CT evidence of acute } \\
\text { exacerbation }\end{array}$ & 4 \\
\hline
\end{tabular}


Fig 1: CT of a 33 year old male with acute pancreatitis showing bulky and heterogenous tail of the pancreas with peripancreatic fat stranding

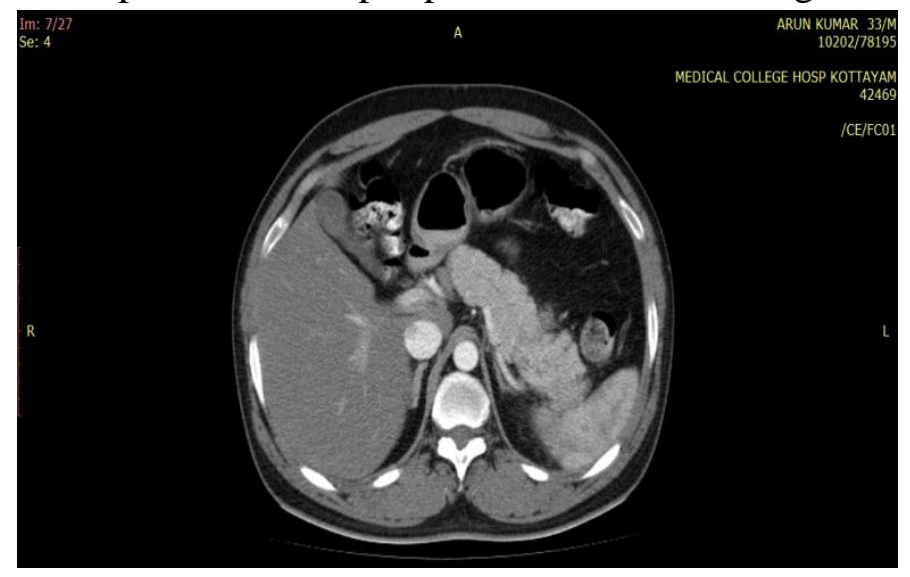

Fig 2: CT of a 56 year old male with acute pancreas showing more than thirty percent pancreatic necrosis. Air pocket noted within, suggestive of infected necrosis

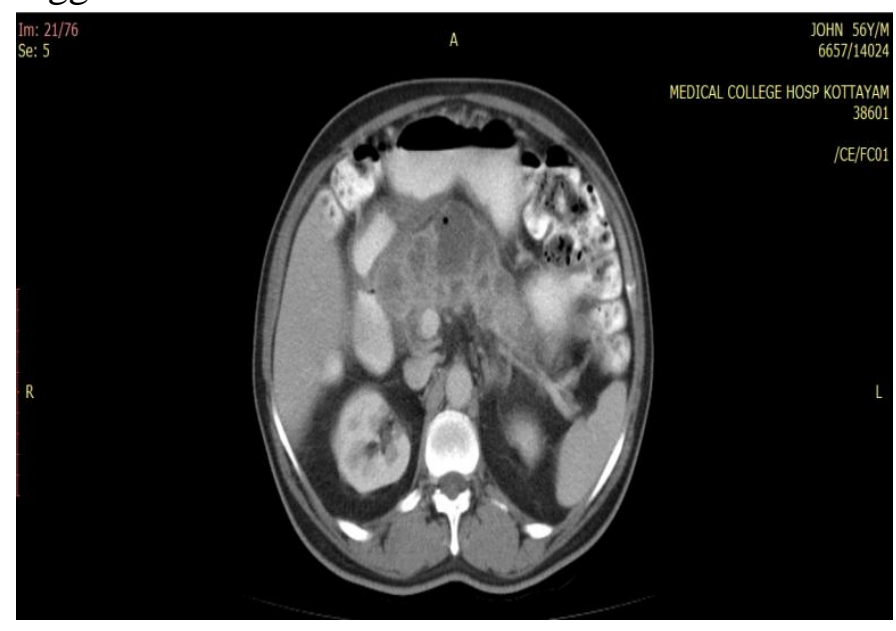

\section{Discussion}

In our study peripancreatic fat strandings were seen in $80 \%$ of the cases, making it the most common finding followed by increase in pancreatic size (78\%). 39\% of the patients had fluid collection, most of which is extrapancreatic fluid collection.

Pancreatic necrosis was seen in $15 \%$ of the cases ( $11 \%$ of the patients had less than $30 \%$ necrosis and $5 \%$ of the patients had more than $30 \%$ necrosis). Peripancreatic fat necrosis was seen in only 6 cases. Studies conducted by Kemppainen E et $\mathrm{al}^{8}$ found $10-20 \%$ patients of acute pancreatitis develop necrosis.
Extrapancreatic involvement was seen in $56 \%$ of the cases. The most common extrapancreatic involvement was ascites, which was seen in $46 \%$ of cases, followed by pleural effusion ( $16 \%$ of the cases) and gastrointestinal involvement (15\% of the cases).

Balthazar $\mathrm{EJ}^{9}$, In his first study in 1985, graded acute pancreatitis into five distinct groups (from A to E), and attempted to correlate the computed tomography grade with clinical follow up findings, morbidity, and mortality. He reviewed in 2002, to describe the accepted new concepts in the development of pancreatic necrosis and staging of acute pancreatitis. The computed tomography severity index helps in development and extent of pancreatic necrosis as it is important indicators for disease severity. They concluded by saying contrast-enhanced computed tomography is the imaging modality of choice to stage the severity of inflammatory process, detect pancreatic necrosis and depict local complications. It yielded overall detection rate of $90 \%$ with close to $100 \%$ sensitivity after 4days for pancreatic gland necrosis.

Using modified CT severity index score, Maximum number of patients had a total score of $4(27 \%)$ followed by score $6(26 \%)$. Least number of patients had score $10(1 \%) .12 \%$ of cases had score 0 .

Based on the scores obtained, patients were classified to have mild, moderate and severe disease. Patient with score $0-2$ were classified as having mild disease, $4-6$ as having moderate disease, $8-10$ as having severe disease. Maximum number of cases in our study were found to have moderate disease (53\% of cases) followed by mild disease ( $36 \%$ of cases). Severe pancreatitis was seen in only $11 \%$ of cases. In a similar study conducted by Mortele et al with a sample of $66,34(51 \%)$ had mild pancreatitis, 22 (33\%) had moderate pancreatitis and $10(15 \%)$ had severe pancreatitis. ${ }^{10}$

$1.9 \%$ of moderate pancreatitis and $27 \%$ of severe pancreatitis patients died. 


\section{Conclusion}

Computed tomography plays a very important role in management of patients with acute pancreatitis, complementing laboratory investigations such as serum amylase and lipase level that have high sensitivity and specificity. Computed tomography is of great use to grade the extent and severity of the acute pancreatitis. Prognosis of acute pancreatitis can be depicted by Modified CT severity index. Computed tomography is also very useful in the early detection of complications of acute pancreatitis.

\section{References}

1. Tulp N. Observationum medicarum. 2nd ed. Amsterdam, The Netherlands: Editio Nova et Aucta; 1652.

2. Tinto A, Lloyd D A, Kangjy et al. Acute and chronic pancreatitis - disease on the rise: A study of hospital admissions in England. Alimentary pharmacology and therapy. 2002: p. 2097-2105.

3. Frey C F, Zhou H, Harvey D J, White R H. The incidence and case fatality rates of acute biliary, alcoholic and idiopathic pancreatitis in california 1994-2001. Pancreas. 2006: p. 336-344.

4. Merkele E M, Gorich J. Imaging of Acute pancreatitis. European Radiology.2002;12: P.1979-1992.

5. Clavien $\mathrm{P} A$, hauser $\mathrm{H}$, Meyer $\mathrm{P}$, et al. Value of contrast enhanced CT in the early diagnosis \& prognosis of acute pancreatitis. American journal of surgery. 1988;(155): p. 457 - 466.

6. Mortele K J, P A Banks, S G Silverman. State of art imaging of Acute pancreatitis. JBR-BTR. 2003;(86): p. 193-208.

7. Mortele et al. A modified CT severity index for evaluating acute pancreatitis: improved correlation with patient outcome. American journal of roentgenology. 2004: p. 1261-1265.

8. Kemppainen E, Sainio V, Haapiainen R,
Kivisaari L, Kivilaakso E, Poolakkainen P. Early localisation of necrosis by contrast enhanced computed tomography can predict outcome in severe acute pancreatitis. British journal of surgery. 1996 july: p. 924-929..

9. Balthazar E J. Acute pancreatitis: Assessment of severity with clinical and CT evaluation. Radiology. 2002 June: p. 603-613.

10. Mortele K J, Merge P, Taylor H, Ernst M, Ros P R. Renal and perirenal space involvement in acute pancreatitis: state of the art spiral CT findings. Abdominal Imaging. 2000: p. 272-278. 